\title{
Case Report \\ Beneficial Effect of Conversion to Belatacept in Kidney-Transplant Patients with a Low Glomerular-Filtration Rate
}

\author{
Julie Belliere, ${ }^{1}$ Céline Guilbeau-Frugier, ${ }^{2,3}$ Arnaud Del Bello, ${ }^{1}$ \\ Laure Esposito, ${ }^{1}$ Caroline Capuani, ${ }^{2,3}$ Isabelle Cardeau-Desangles, ${ }^{1}$ \\ Lionel Rostaing, ${ }^{1,2,4}$ and Nassim Kamar ${ }^{1,2,4}$ \\ ${ }^{1}$ Department of Nephrology and Organ Transplantation, CHU Rangueil, TSA 50032, 31059 Toulouse Cedex 9, France \\ ${ }^{2}$ Université Paul Sabatier, 31062 Toulouse, France \\ ${ }^{3}$ Department of Pathology, CHU Rangueil, 31059 Toulouse, France \\ ${ }^{4}$ INSERM U1043, IFR-BMT, CHU Purpan, 31059 Toulouse, France
}

Correspondence should be addressed to Nassim Kamar; kamar.n@chu-toulouse.fr

Received 11 March 2014; Revised 28 April 2014; Accepted 30 April 2014; Published 18 May 2014

Academic Editor: Mariano Ferraresso

Copyright (C) 2014 Julie Belliere et al. This is an open access article distributed under the Creative Commons Attribution License, which permits unrestricted use, distribution, and reproduction in any medium, provided the original work is properly cited.

\begin{abstract}
Belatacept has been found to be efficient at preserving good kidney function in maintenance kidney-transplant patients. Herein, we report on the use of belatacept as a rescue therapy for two kidney-transplant patients presenting with severe adverse events after treatment with calcineurin inhibitors (CNIs) and mammalian target-of-rapamycin (mTOR) inhibitors. Two kidney-transplant patients developed severely impaired kidney function after receiving CNIs. The use of everolimus was associated with severe angioedema. Belatacept was then successfully used to improve kidney function in both cases, even though estimated glomerularfiltration rate before conversion was $<20 \mathrm{~mL} / \mathrm{min}$. These case reports show that belatacept can be used as a rescue therapy, even if kidney function is very low in kidney-transplant patients who cannot tolerate CNIs and/or mTOR inhibitors.
\end{abstract}

\section{Introduction}

Interstitial fibrosis and tubular atrophy (IFTA) are two of the major causes of graft loss after kidney transplantation. Calcineurin inhibitors (CNIs) are well known to have nephrotoxic effects on the kidney allograft, leading to IFTA and graft loss [1]. Mammalian target-of-rapamycin (mTOR) inhibitors, such as sirolimus and everolimus, have been used in CNI-free regimens or in association with low-dose CNIs to reduce CNI dosage and, thus, nephrotoxicity [2]. However, although mTOR-based immunosuppression regimens can improve kidney function and reduce IFTA, their safety profile remains worrisome [2]. Indeed, their side effects are often unpredictable and lead to interruption of treatment in $40 \%$ of cases [3]. Hence, in some situations, patients can be intolerant and/or contraindicated to the large majority of immunosuppressive drugs. Consequently, preserving graft function and avoiding acute rejection then become a medical challenge.
Recently, belatacept (CTLA4-Ig) has been developed to block CD80/86 and thereby inhibit T-cell costimulation [4, 5]. Two phase-III trials have compared the efficacy and safety of belatacept to that of cyclosporine A in association with mycophenolate mofetil (MMF) and steroids in de novo kidney-transplant patients who had received a kidney allograft from standard- and extended-criteria donors. In belatacept-treated patients, although the incidence of acute rejection was slightly higher, long-term kidney function was significantly improved [6-9]. In addition, tolerance to belatacept was excellent.

Another phase-III study has assessed the effect of converting from CNIs (cyclosporine A or tacrolimus) to belatacept. Kidney-transplant patients, who had an estimated glomerular-filtration rate (using the MDRD equation) of between 35 and $75 \mathrm{~mL} / \mathrm{min}$, were randomized to be either maintained on CNIs or were converted to belatacept [10, 11]. The data collected over 3 years showed significantly better 


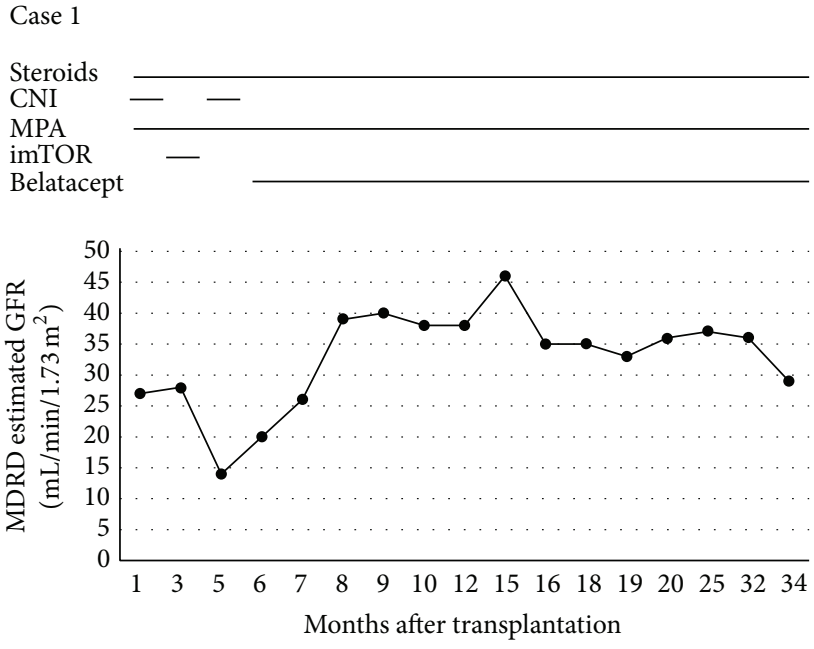

(a)
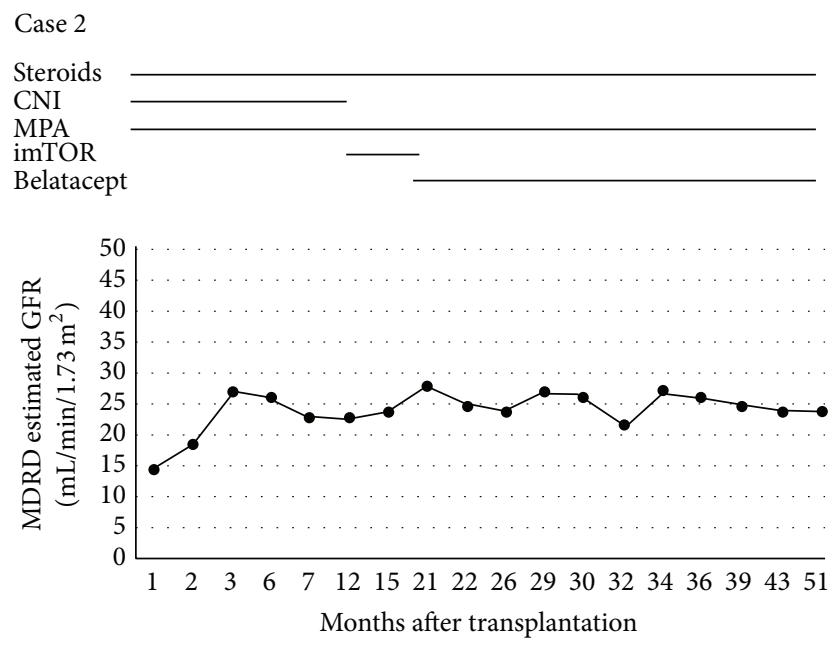

(b)

FIGURE 1: Kidney function. Glomerular-filtration rate (GFR) values were estimated with MDRD and reported for each case according to the time after transplantation. CNI: calcineurin inhibitors; MPA: mycophenolic acid; imTOR: mTOR (mammalian target of rapamycin) inhibitors.

kidney function in patients converted to belatacept compared to those receiving CNIs (either tacrolimus or cyclosporine A) [12]. The effect of conversion from CNIs to belatacept, as a rescue therapy for kidney-transplant patients with a glomerular-filtration rate (GFR) of $<35 \mathrm{~mL} / \mathrm{min}$, is unknown.

Herein, we describe two kidney-transplant recipients with severe intolerance to CNIs and mTOR inhibitors who were successfully converted to belatacept. Glomerular-filtration rate (GFR) values are reported for each case in Figure 1.

\section{Cases Reports}

The patients' and donors' characteristics are presented in Table 1.

Case 1. A 52-year-old woman received a second kidney allograft for lupus nephritis and antiphospholipid antibody syndrome. The initial immunosuppressive therapy included basiliximab, tacrolimus, mycophenolic acid (MPA), and steroids. At one month after transplantation, because of persisting impaired kidney function (creatinine level $171 \mu \mathrm{mol} / \mathrm{L}$, eGFR of $27 \mathrm{~mL} / \mathrm{min} / 1.73 \mathrm{~m}^{2}$ ), a kidney biopsy was performed and showed ischemic tubular necrosis and signs of severe nephroangiosclerosis (t0 i0 g0 v0 ptc0 ah0 cg0 ci0 ct0 cv2 $\mathrm{mm} 0$, according to the Banff classification [13]), which was attributed to the donor, that is, a 77-year-old woman with a history of hypertension and who had died from a stroke. At that time, in order to avoid tacrolimus-induced nephrotoxicity, tacrolimus was replaced by everolimus. Kidney function remained unchanged. However, a switch back from everolimus to tacrolimus (target trough level 3-5 ng/mL) was done 2 months later because of severe angioedema. By five months after transplantation, the patient presented with heart failure, which was related to severe mitral-valve disease; thus, heart surgery was performed. After surgery, she developed acute kidney failure, which required dialysis for 6 days. Thereafter, her kidney function slightly improved but remained at $\sim 400 \mu \mathrm{mol} / \mathrm{L}\left(\mathrm{eGFR}\right.$ of $10 \mathrm{~mL} / \mathrm{min} / 1.73 \mathrm{~m}^{2}$ ). Hence, in order to avoid CNI-induced nephrotoxicity, tacrolimus was replaced by belatacept $(5 \mathrm{mg} / \mathrm{kg} / \mathrm{month})$ at 6 months after transplantation, plus MPA ( $2 \mathrm{~g} / \mathrm{d})$ and steroids ( $5 \mathrm{mg} /$ day $)$. By 12 months after the switch, serum-creatinine level was $138 \mu \mathrm{mol} / \mathrm{L}$ and eGFR was $35 \mathrm{~mL} / \mathrm{min} / 1.73 \mathrm{~m}^{2}$.

At the last follow-up, that is, 29 months after the switch, serum-creatinine level and eGFR were, respectively, $162 \mu \mathrm{mol} / \mathrm{L}$ and $29 \mathrm{~mL} / \mathrm{min} / 1.73 \mathrm{~m}^{2}$. Proteinuria, which was $80 \mathrm{mg} / \mathrm{g}$ of creatinine before the switch, had decreased to $18 \mathrm{mg} / \mathrm{g}$ of creatinine at the last follow-up. Tolerance to the immunosuppressive treatment was excellent. No acuterejection episode occurred and no donor-specific anti-HLA antibodies (DSAs) were detected using the Luminex singleantigen assay before or after the switch to belatacept.

Case 2. A 69-year-old man received a first kidney allograft for vascular nephropathy from an extended-criteria donor. The donor was a 74-year-old man with a history of diabetes and hypertension. The initial immunosuppressive therapy was based on basiliximab, tacrolimus, MPA, and steroids. After transplantation, his kidney function remained very poor. eGFR was 15,26 , and $23 \mathrm{~mL} / \mathrm{min} / 1.73 \mathrm{~m}^{2}$ at 1,6 , and 12 months, respectively. A kidney biopsy, performed at 3 months posttransplantation, revealed signs of diabetic nephropathy and mild tubulopathy, but no rejection (t0 i0 g0 v0 ptc0 ah3 cg0 cil ctl cvl mml, according to the Banff classification). At one year, tacrolimus was replaced by everolimus. Consequently, he presented with severe dyslipidemia and anemia, which required large doses of erythropoietin. In addition, steroid dose had to be reduced because of bilateral femoral osteonecrosis.

Another kidney biopsy was performed 6 months later, which showed grade-2 IFTA and severe vascular lesions (cv3, 
TABLE 1: Donors' and recipients' characteristics.

\begin{tabular}{lcc}
\hline & Case 1 & Case 2 \\
\hline Donor & 77 & \\
Age (years) & $\mathrm{W}$ & 60 \\
Gender & 25.4 & 29 \\
Body mass index $\left(\mathrm{kg} / \mathrm{m}^{2}\right)$ & Hemorrhagic & Ischemic \\
Cause of death & stroke & stroke \\
Cardiac arrest & no & no \\
Serum creatinine level $(\mu \mathrm{mol} / \mathrm{L})$ & 52 & 71 \\
Proteinuria $(\mathrm{g} / \mathrm{L})$ & 0.12 & 0.17 \\
Estimated MDRD GFR & 100 & 74 \\
(mL/min/1.73 m $\left.{ }^{2}\right)$ & & \\
Recipient & 52 & 69 \\
Age (years) & W & M \\
Gender & 26.4 & 24.4 \\
Body mass index $\left(\mathrm{kg} / \mathrm{m}^{2}\right)$ & Lupus & Vascular \\
Cause of kidney disease & 765 & 690 \\
Cold ischemia time $(\mathrm{min})$ & 45 & 60 \\
Warm ischemia time $(\mathrm{min})$ &
\end{tabular}

W: woman; M: male; GFR: glomerular-filtration rate.

according to the Banff classification). Kidney function was altered; serum-creatinine level was $256 \mu \mathrm{mol} / \mathrm{L}$, eGFR was $22 \mathrm{~mL} / \mathrm{min} / 1.73 \mathrm{~m}^{2}$, and proteinuria was $1.5 \mathrm{mg} / \mathrm{g}$ of creatinine. At that time, that is, 19 months after transplantation, everolimus was replaced by belatacept $(5 \mathrm{mg} / \mathrm{kg} / \mathrm{month})$. MPA (2g/d) and low-dose steroids $(5 \mathrm{mg} / \mathrm{d})$ were continued. At the last follow-up, that is, 33 months after the switch, serum-creatinine level was $224 \mu \mathrm{mol} / \mathrm{L}$, eGFR was $24 \mathrm{~mL} / \mathrm{min} / 1.73 \mathrm{~m}^{2}$, and proteinuria had decreased to $0.7 \mathrm{mg} / \mathrm{g}$. Metabolic and hematological parameters were both improved. No DSAs were detected before or after the switch to belatacept.

\section{Discussion}

Within the last decade, the number of extended-criteria donors has increased. Consequently, for a large number of kidney-allograft recipients, the use of CNIs, which are known to have nephrotoxic effects, can be problematic. In de novo kidney-transplant patients who receive a kidney from an extended-criteria donor, the use of belatacept has been associated with significantly better kidney function at 5 years compared to patients that received cyclosporine A [6]. In maintenance kidney-transplant patients with preserved kidney function (eGFR between 35 and $75 \mathrm{~mL} / \mathrm{min}$ ), conversion from CNIs to belatacept significantly improved kidney function compared to those maintained on CNIs [10-12]. However, the effect of belatacept on kidney function in patients with impaired kidney function, that is, eGFR $<35 \mathrm{~mL} / \mathrm{min}$, is unknown.
mTOR inhibitors have been used in conversion protocols to avoid CNI-induced nephrotoxicity [2]. However, late conversion from CNIs to mTOR inhibitors, when eGFR is $<30 \mathrm{~mL} / \mathrm{min}$ and/or when proteinuria is $>0.5 \mathrm{mg} / \mathrm{g}$ of creatinine, does not prevent a decline in kidney function $[14,15]$. In addition, mTOR inhibitors have several side effects that result in a high rate of treatment withdrawal, that is, $40 \%$ [3].

Herein, we have described two kidney-transplant recipients who were intolerant to both CNIs and mTOR inhibitors. The two kidney-transplant patients had severe impaired kidney function because of severe histological lesions related to the donor. The use of CNIs led to very low eGFR $(<20 \mathrm{~mL} / \mathrm{min})$. The use of everolimus was associated with severe angioedema, requiring its withdrawal. Hence, belatacept was successfully used and led to improved kidney function in both cases, even though eGFR before conversion was $<20 \mathrm{~mL} / \mathrm{min}$. Neither of the patients developed a serious adverse event, donor-specific antibodies, or posttransplant lymphoma disease.

In conclusion, these case reports highlight the fact that belatacept can be used as a rescue therapy, even if kidney function is very low, in kidney-transplant patients who cannot tolerate CNIs and/or mTOR inhibitors.

\section{Conflict of Interests}

The authors declare that there is no conflict of interests regarding the publication of this paper.

\section{Authors' Contribution}

Julie Belliere collected the data and wrote the paper. Céline Guilbeau-Frugier and Caroline Capuani did the pathological work-up. Arnaud Del Bello, Laure Esposito, and Isabelle Cardeau-Desangles did the follow-up to patients. Lionel Rostaing designed the study, participated in the patients' followup, and reviewed the paper. Nassim Kamar designed the study, participated in the patients' follow-up, and wrote the paper.

\section{References}

[1] M. Pascual, T. Theruvath, T. Kawai, N. Tolkoff-Rubin, and A. Benedict Cosimi, "Strategies to improve long-term outcomes after renal transplantation," The New England Journal of Medicine, vol. 346, no. 8, pp. 580-590, 2002.

[2] L. Rostaing and N. Kamar, "mTOR inhibitor/proliferation signal inhibitors: entering or leaving the field?" Journal of Nephrology, vol. 23, no. 2, pp. 133-142, 2010.

[3] N. Pallet and C. Legendre, "Adverse events associated with mTOR inhibitors," Expert Opinion on Drug Safety, vol. 12, pp. 177-186, 2013.

[4] C. P. Larsen, T. C. Pearson, A. B. Adams et al., "Rational development of LEA29Y (belatacept), a high-affinity variant of CTLA4Ig with potent immunosuppressive properties," The American Journal of Transplantation, vol. 5, no. 3, pp. 443-453, 2005.

[5] F. Vincenti, C. Larsen, A. Durrbach et al., "Costimulation blockade with belatacept in renal transplantation," New England Journal of Medicine, vol. 353, no. 8, pp. 770-781, 2005. 
[6] B. Charpentier, J. O. Medina Pestana, C. R. M. Del et al., "Longterm exposure to belatacept in recipients of extended criteria donor kidneys," The American Journal of Transplantation, vol. 13, pp. 2884-2891, 2013.

[7] A. Durrbach, J. M. Pestana, T. Pearson et al., "A phase III study of belatacept versus cyclosporine in kidney transplants from extended criteria donors (BENEFIT-EXT Study)," The American Journal of Transplantation, vol. 10, no. 3, pp. 547-557, 2010.

[8] L. Rostaing, F. Vincenti, J. Grinyo et al., "Long-term belatacept exposure maintains efficacy and safety at 5 years: results from the long-term extension of the BENEFIT study," The American Journal of Transplantation, vol. 13, pp. 2875-2883, 2013.

[9] F. Vincenti, B. Charpentier, Y. Vanrenterghem et al., "A phase III study of belatacept-based immunosuppression regimens versus cyclosporine in renal transplant recipients (BENEFIT Study)," The American Journal of Transplantation, vol. 10, no. 3, pp. 535$546,2010$.

[10] J. Grinyó, B. Charpentier, J. M. Pestana et al., "An integrated safety profile analysis of belatacept in kidney transplant recipients," Transplantation, vol. 90, no. 12, pp. 1521-1527, 2010.

[11] L. Rostaing, P. Massari, V. D. Garcia et al., "Switching from calcineurin inhibitor-based regimens to a belatacept-based regimen in renal transplant recipients: a randomized phase II study," Clinical Journal of the American Society of Nephrology, vol. 6, no. 2, pp. 430-439, 2011.

[12] N. Kamar, J. Alberu, S. Steinberg et al., "3-year outcomes after switching to belatacept from a CNI in stable kidney transplant recipients," in Proceedings of the 16th Conference of the European Society for Organ Transplantation, Vienna, Austria, September.

[13] B. Sis, M. Mengel, M. Haas et al., "Banff'09 meeting report: antibody mediated graft deterioration and implementation of Banff working groups," The American Journal of Transplantation, vol. 10, no. 3, pp. 464-471, 2010.

[14] F. Diekmann, K. Budde, F. Oppenheimer, L. Pritsche, H. H. Neumayer, and J. M. Campistol, "Predictors of success in conversion from calcineurin inhibitor to sirolimus in chronic allograft dysfunction," The American Journal of Transplantation, vol. 4, no. 11, pp. 1869-1875, 2004.

[15] A. I. Sánchez-Fructuoso, J. C. Ruiz, N. Calvo et al., "Everolimus as primary immunosuppression in kidney transplantation: experience in conversion from calcineurin inhibitors," Transplantation, vol. 93, no. 4, pp. 398-405, 2012. 


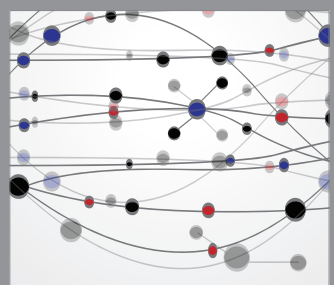

The Scientific World Journal
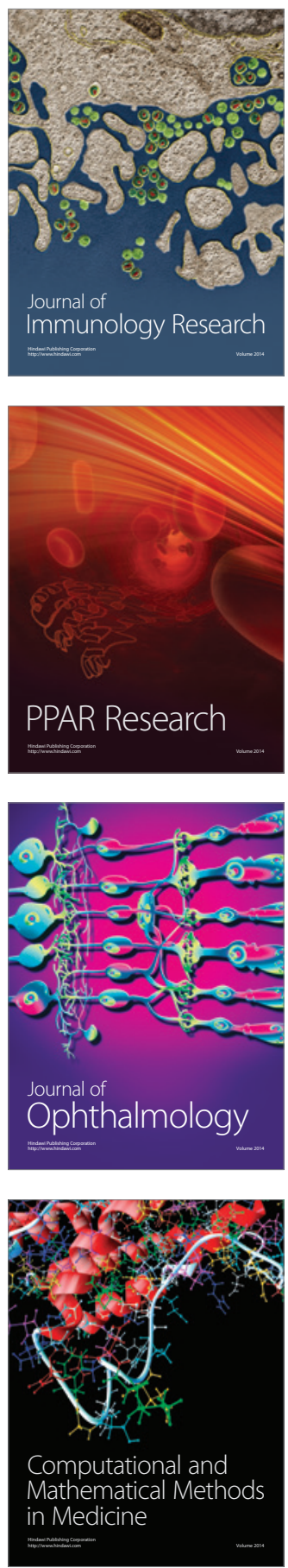

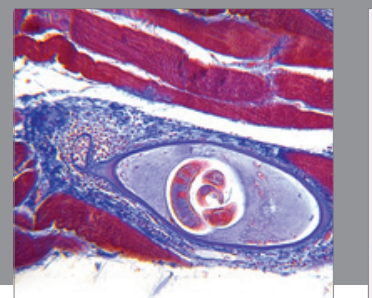

Gastroenterology

Research and Practice
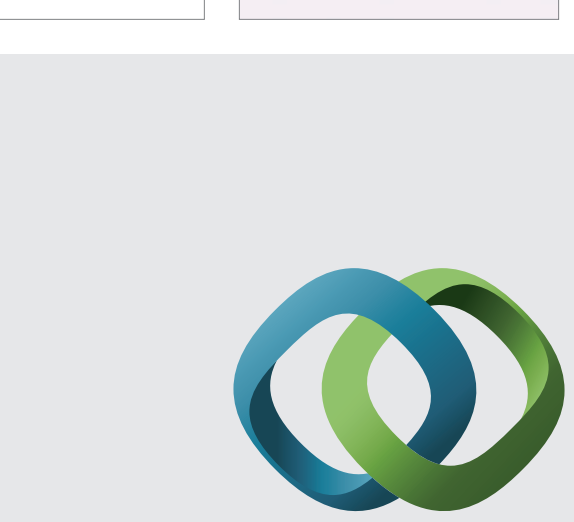

\section{Hindawi}

Submit your manuscripts at

http://www.hindawi.com
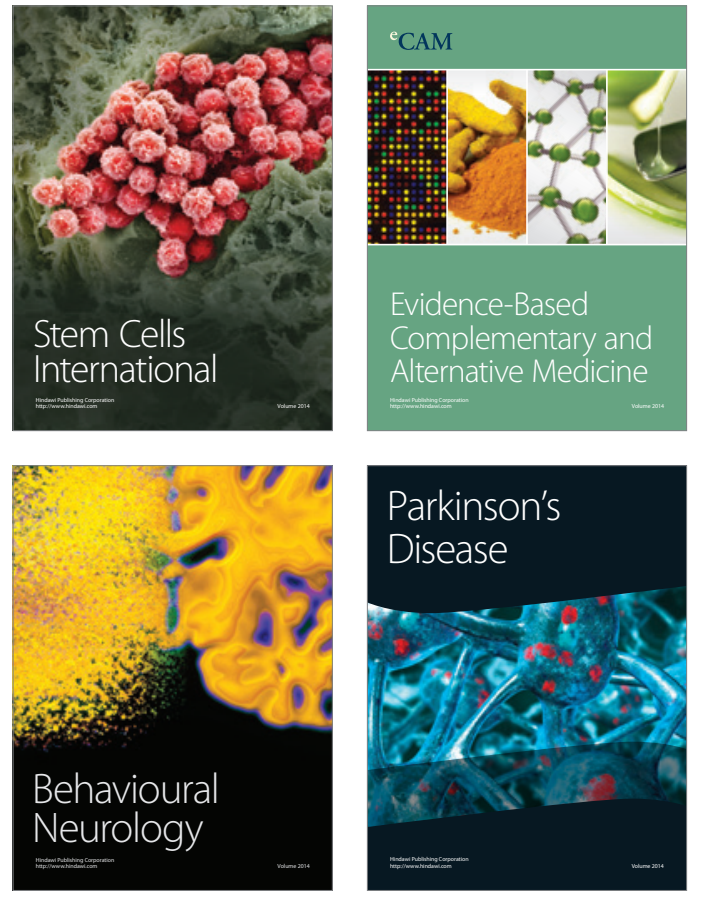
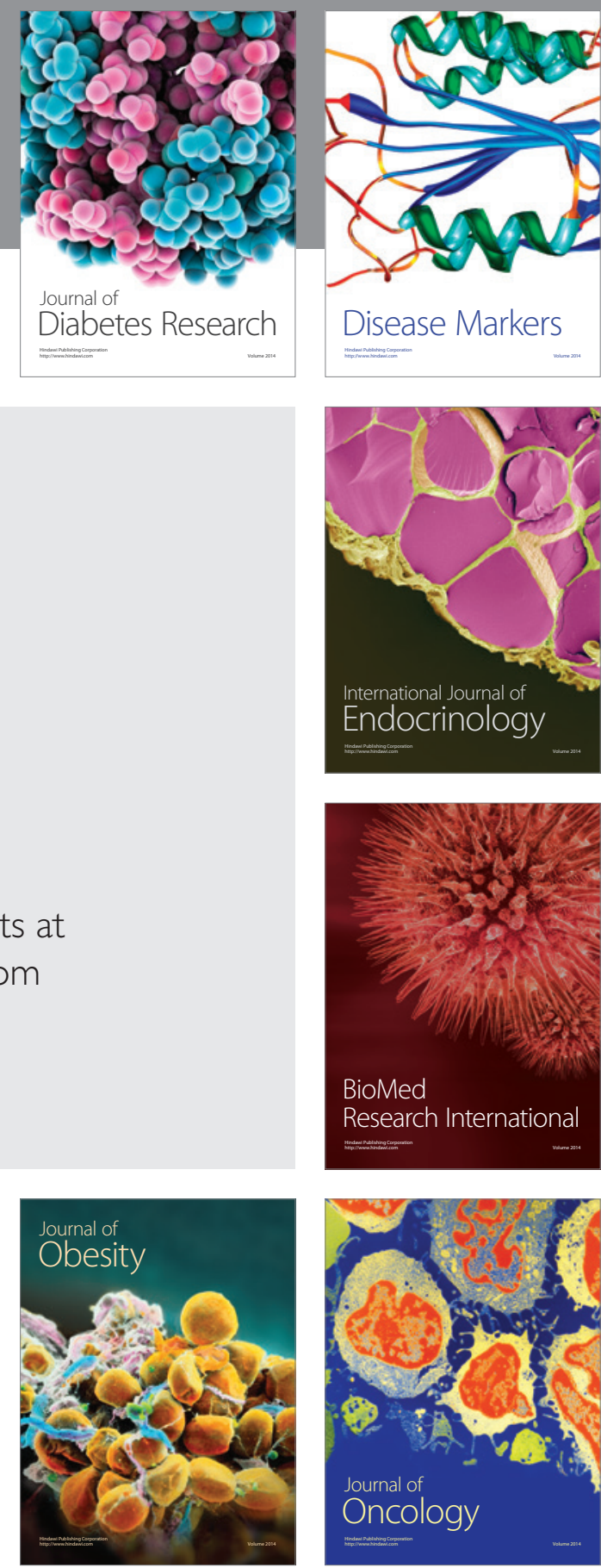

Disease Markers
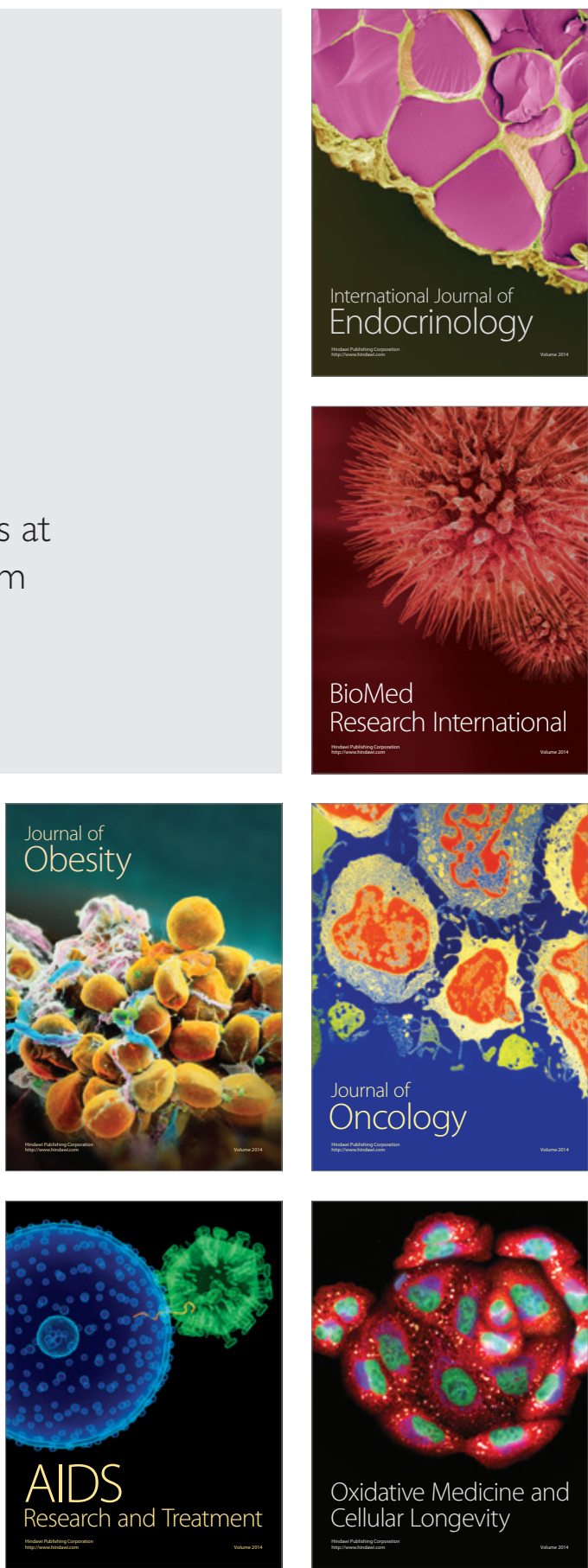